\title{
0988 SUICIDE - CURRENT MAZE AND FUTURE MOVEMENT
}

L Vijayakumar* Correspondence: Department of Psychiatry, Voluntary Health Services, 25, Ranjith Road, Kotturpuram, Chennai 600 085, India

10.1136/ip.2010.029215.988

There are a million deaths by suicide every year and by 2020 it is expected to be 1.53 million. The global burden of disease by suicide is also expected to increase from $1.8 \%$ to $2.4 \%$ signifying that suicide is a major public health problem. Around $60 \%$ of suicides occur in Asia. Suicide is a complex human behaviour resulting from a maze of biological, psychological, socio cultural and environmental risk and protective factors that interact intricately. The resulting behaviour varies in patterns and the responses it elicits in different countries and regions. In developed countries, the male to female ratio for suicide is between 2 and 4 to 1 . Asian countries, show much lower male to female ratio. Mental disorders occupy a premier position in the matrix of causation of suicide, however in developing countries like China and India, social and environmental factors appear more significant. Exposure to violence and Intimate partner violence is also strongly linked to suicide. Worldwide, suicide by ingestion of pesticides is the commonest method accounting for $30 \%$ of suicides. It is more so in rural areas of the developing world. Hence, restriction of access to pesticide through safer individual and community storage and stopping sales of more toxic preparations has the potential to reduce suicide. A $10 \%$ reduction in pesticide suicide would result in 30 000 lives saved. Several developed countries have established national suicide prevention programmes, but their effectiveness has not been conclusively proven. The enormity of the problem needs a global movement towards significant reduction of suicides. Suicide prevention should not be viewed as a traditional exercise in health sector but as a social objective. 